\title{
Effectiveness of the Capparis spinosa Treatment in Vitiligo
}

Nazan EMIROĞLU, Fatma Pelin CENGiZ, Anil GÜLSEL BAHALI, Dilek BIYIK ÖZKAYA, Özlem SU, Nahide ONSUN

Department of Dermatology, Bezmialem Vakif University School of Medicine, İstanbul, Turkey

\section{ABSTRACT}

Objective: We aim to compare the efficacy of topical steroids and Capparis spinosa extract in the treatment of vitiligo.

Methods: Patients were retrospectively identified from those treated by the Department of Dermatology. 60 vitiligo patients were included in the study. 30 of them were given topical steroids, and 30 of them were treated with Capparis spinosa extract. VASI (Vitiligo Area Scoring Index) were calculated statistically before treatment and during the sixth month of treatment.

Results: Although the VASI value of patients who treated with topical corticosteroids decreased statistically, no statistically significant changes were observed in patients who treated with with Capparis spinosa extract. After treatment, VASI values were significantly lower in patients with treated topical corticosteroids than treated with Capparis spinosa extract $(\mathrm{p}<0.05)$.

Conclusion: In this study, topical corticosteroids were more effective than Capparis spinosa in the treatment of vitiligo. Although there was not statistically difference of VASI values in patients treated with Capparis spinosa. repigmentation was observed after treatment. Therefore Capparis spinosa is not enough alone but maybe it can be used in combination therapy of vitiligo.

Keywords: Vitiligo, treatment, Capparis spinosa

\section{Introduction}

Vitiligo is an acquired disease with an unknown etiology, in which losses appear in the functional melanocytes of the skin, which is clinically depigmented and progresses with macules and patches. Although it has many treatment options with an incidence of approximately $0.5 \%-2 \%$, its efficacy is very limited, and response to treatment varies from patient to patient (1). Treatment failures have led to new treatment needs. Experimental treatment methods are important in this point.

Topical and systemic therapies, phototherapy, depigmenting agents, surgical procedures, or combinations of these treatments are used in patients with vitiligo $(2,3)$.

The aim of the present study was to compare the efficacy of Capparis spinosa extract with the efficacy of topical steroid therapy in vitiligo in which some patients are still resistant to treatment despite many treatment options, requiring new treatment options.

\section{Methods}

Treatment of patients with vitiligo who were examined between January 2014 and July 2015 in the dermatology polyclinic of our hospital was retrospectively screened. The ethics committee approved the study. The study was conducted in accordance with the Declaration of Helsinki (1964), which was corrected in 1996 at Somerset West.

Patients were divided into two groups. Patients with localized vitiligo who were treated with topical C. spinosa were classified as the patient group, and patients with localized vitiligo who were treated with topical corticosteroids were classified as the control group. Sixty adult patients clinically diagnosed with vitiligo were included in the study. Pregnant and 
breastfeeding patients and patients using systemic immunosuppressives, any topical drug, or other drugs for vitiligo were excluded from the study. Vitiligo Area Scoring Index (VASI) was calculated using photographs used in the clinical followups of the patients. Written informed consent was obtained from the patients.

VASI is recommended to assess the severity of vitiligo. The extent of lesion and the degree of depigmentation are the parameters assessed using VASI. The extent of the affected area is calculated by assuming that the palm (palmar area+volar face of the fingers) corresponds to $1 \%$ body surface area. The degree of depigmentation is expressed as $0 \%, 25 \%, 50 \%, 75 \%$, and $100 \%$ according to the pigment ratio in the lesion. Full depigmentation is considered as $100 \%$. VASI is calculated by the formula: lesion extentxdepigmentation degree. It is a scoring used to assess the response to treatment. In the present study, VASI of the patients who were treated with topical corticosteroids and topical C. spinosa was calculated before treatment and 6 months of treatment and was compared statistically. Statistical analysis was assessed using the $\chi^{2}$ test. A p value $<0.05$ was considered significant.

\section{Results}

Sixty patients with vitiligo were included in the study. Topical C. spinosa was initiated in 30 patients, and topical corticosteroid was initiated in 30 patients. However, 9 patients who received topical C. spinosa treatment and 10 patients who received topical corticosteroid treatment were excluded from the study because they did not come for the controls or used the medication irregularly. Of the patients who received topical C. spinosa treatment, $12(57.1 \%)$ were females, and 9 $(42.9 \%)$ were males. Of the patients who were included in the study and received topical corticosteroid treatment, 8 (40\%) were females, and $12(60 \%)$ were males. The mean ages of the patient group and the control group were $28.75 \pm 1.29$ and $26.33 \pm 2.13$ years, respectively. There was no statistical difference in terms of age and gender between the patient and control groups.

Only patients with focal vitiligo were included in the study. Response to treatment was assessed through clinical observation. VASI of the patients was calculated.

Although repigmentation was observed after treatment in comparison to before in 7 out of 21 patients who were treated with topical C. spinosa (Figure 1a-d), there was no statistically significant change in their VASI $(\mathrm{p}>0.05)$ (Table 1$)$.

Although repigmentation was observed after treatment in comparison to before in 14 out of 20 patients who received topical corticosteroid, there was a statistically significant decrease in their VASI $(\mathrm{p}<0.05)$ (Table 2, Figure 2a, b).

The VASI scores of the two groups were consistent before treatment. When compared after treatment, the VASI values of the patients who received topical corticosteroid treatment were significantly lower than those who received topical $C$. spinosa treatment $(\mathrm{p}<0.05)$ (Table 1$)$.

\section{Discussion}

Vitiligo is a common disease in the community. It progresses with acquired depigmentation as a result of the dysfunction of the epidermal melanocytes. Its etiology is unknown. It is acquired, idiopathic, clinically depigmented, and characterized by macules. The incidence of vitiligo is approximately $0.5 \%-2 \%$ and usually begins in childhood or in young adult ages (1). Despite the knowledge that genetic, physical, and emotional factors play a role in its etiology, its pathological mechanism is still unknown. Melanocyte loss is observed histopathologically, and three different hypotheses have been proposed as neural, autoimmune, and autocytotoxic in order to explain its pathogenesis $(2,3)$. The most widely accepted hypothesis is autoimmune melanocyte attack and destruction through the cellular immune system. Oxidative stress is based on the autotaxic hypothesis, and it is known that it plays a role in cell death and pathological event (2). In the neural hypothesis, it is suggested that neuropeptides can play a role in the pathogenesis of vitiligo (3).

Treatment of vitiligo varies according to the age of the patient, spread and localization of the lesions, and response to previous treatments. The aim of the treatment is to stop the progression of the disease, provide repigmentation, and slow down the depigmentation process. Repigmentation can be seen spontaneously in $15 \%-25 \%$ of cases (4). Currently, there is no single method to fully treat vitiligo lesions. Of the patients, $15 \%-30 \%$ do not respond to treatment. A hundred percent repigmentation cannot be achieved in vitiligo treatment. On average, $\geq 75 \%$ repigmentation is considered as a perfect response. This response is achieved in $60 \%$ of the patients (5).

Conventional treatment methods include phototherapy, photochemotherapy, and corticosteroids. New treatment methods are immunomodulators (tacrolimus and pimecrolimus), cyclosporin, vitamin D3 analogs, excimer laser, and ultraviolet $\mathrm{B}$ microtherapy. Despite the availability of many treatment modalities, there is no useful method for all patients, and the response to treatment is highly variable. All treatment methods have disadvantages and advantages. For this reason, the treatment choice can vary from patient to patient. In a group of patients, new treatment options are sought because no response to treatment could be achieved $(3,4)$.

Table 1. VASI values before and after treatment

\begin{tabular}{|l|c|c|c|}
\hline Treatment & $\begin{array}{c}\text { Capparis } \\
\text { spinosa }\end{array}$ & $\begin{array}{c}\text { Topical } \\
\text { corticosteroid }\end{array}$ & P \\
\hline Before treatment & $3.22 \pm 2.98$ & $3.46 \pm 3.01$ & $>0.05$ \\
\hline 6 months of treatmerit $3.11 \pm 2.86$ & $1.85 \pm 1.88$ & $<0.05$ \\
\hline \multicolumn{2}{l}{ VASI: Vitiligo Area Scoring Index. Mean \pm SD } & \\
\hline
\end{tabular}




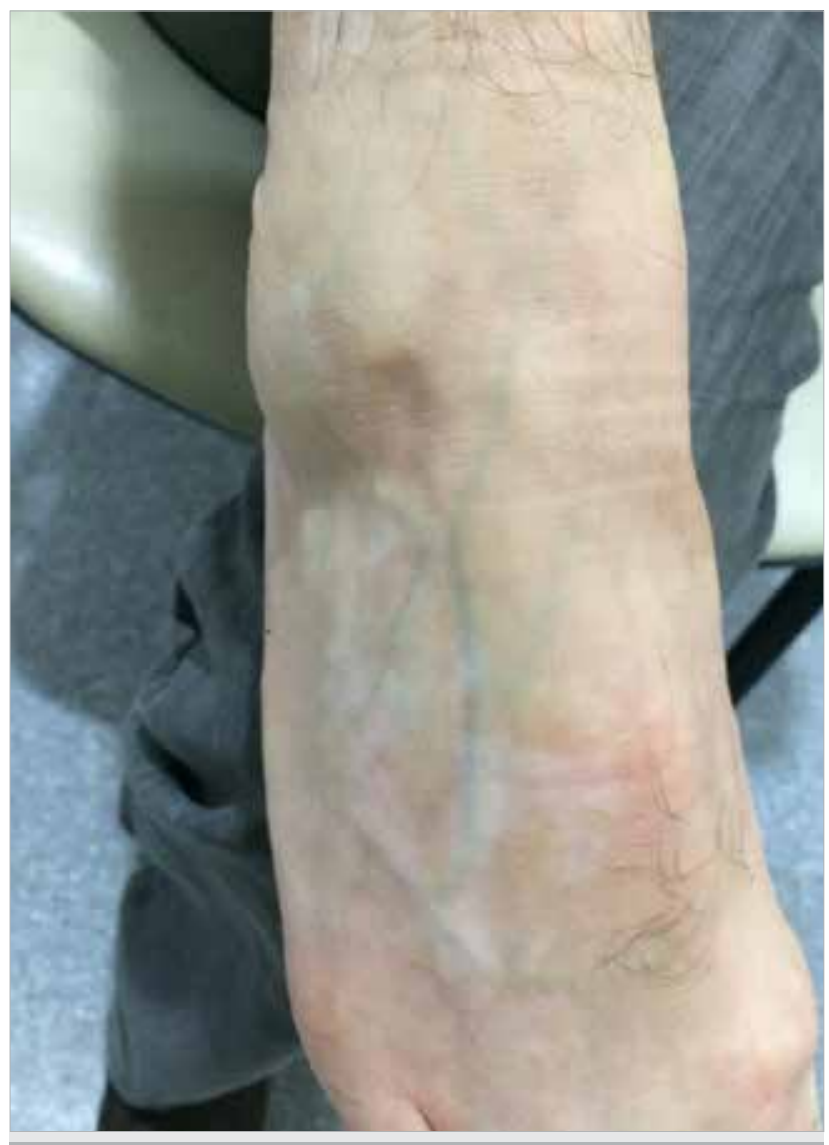

Figure 1. a. Right foot before Capparis spinosa treatment

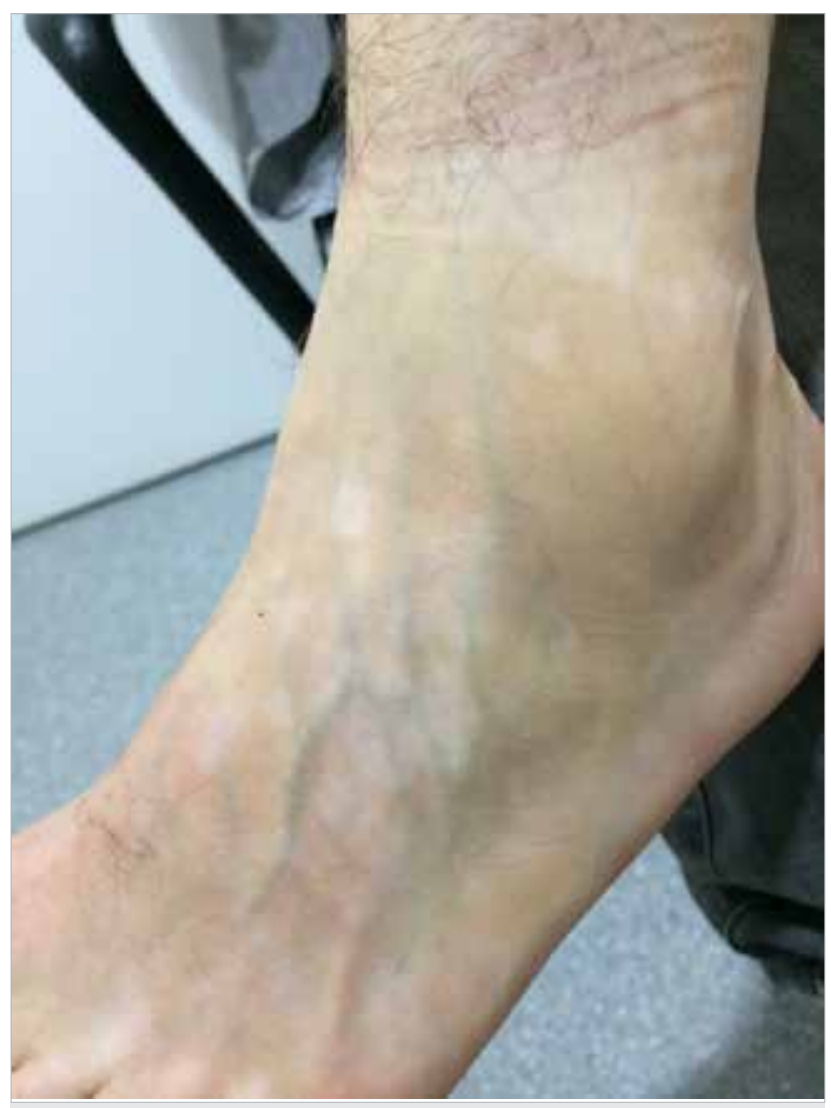

Figure 1. b. Left foot before Capparis spinosa treatment
Experimental treatment methods gain importance at this point. Many treatment methods, such as oral or topical phenylalanine administration, benefiting from the antioxidant effects of alpha-lipoic acid preparations, oral administration of vitamin B12, folic acid, other vitamins and trace elements, use of oral levamisole, use of topical prostaglandin analog, topical application of placental extract (melagenina), topical application of 5-FU, and kheillin+ultraviolet A application, are included in experimental treatments $(6-10)$.

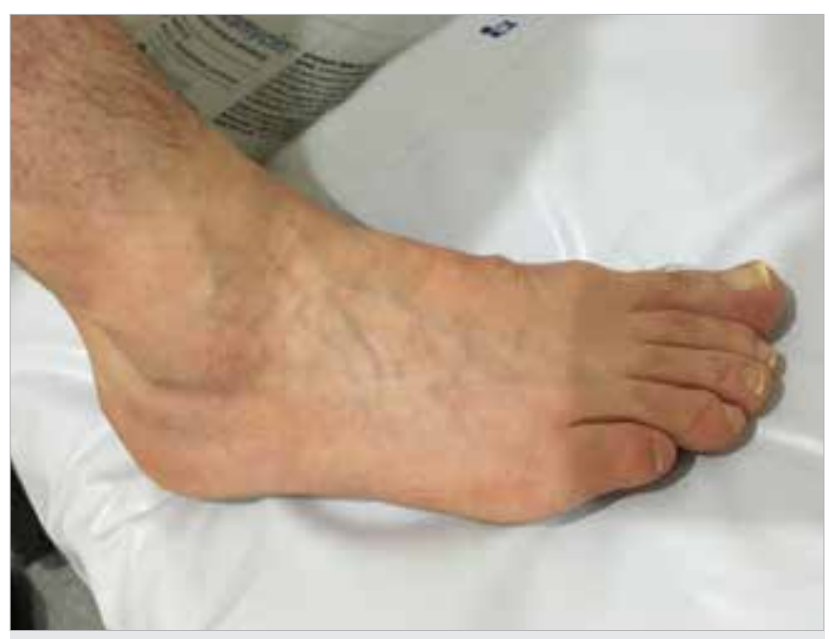

Figure 1. c. Right foot after Capparis spinosa treatment

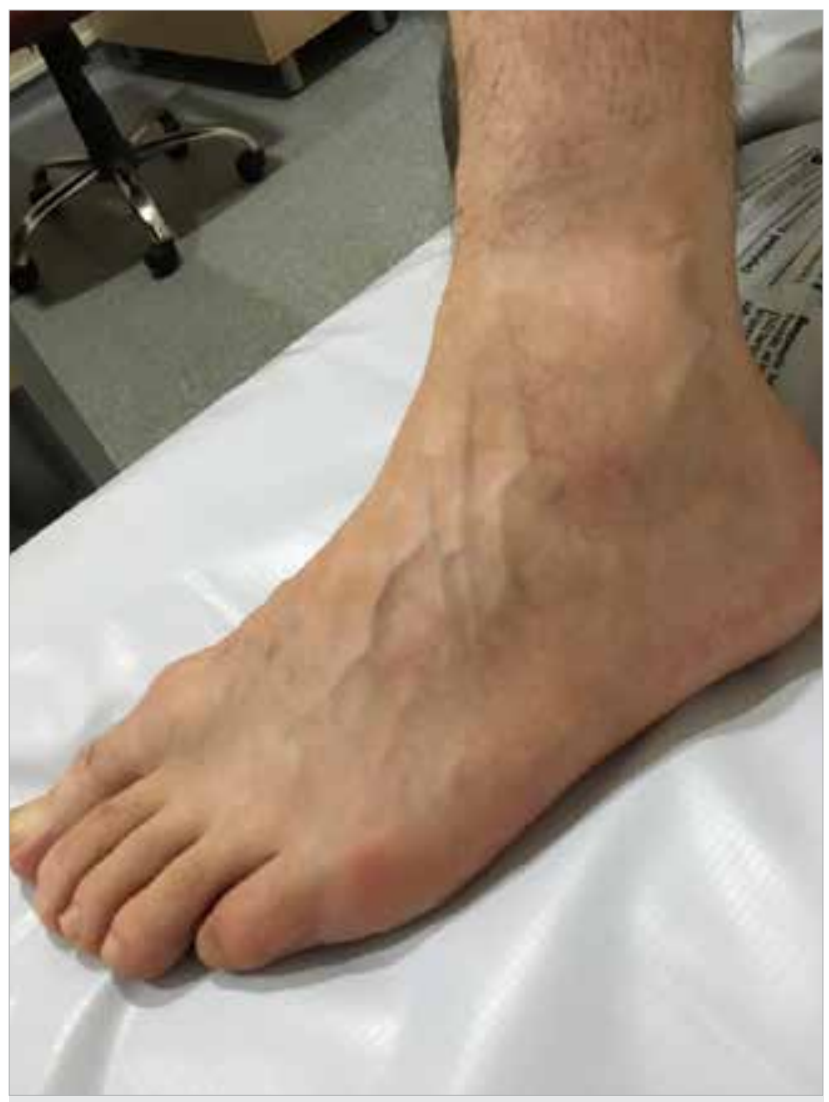

Figure 1. d. Left foot after Capparis spinosa treatment 


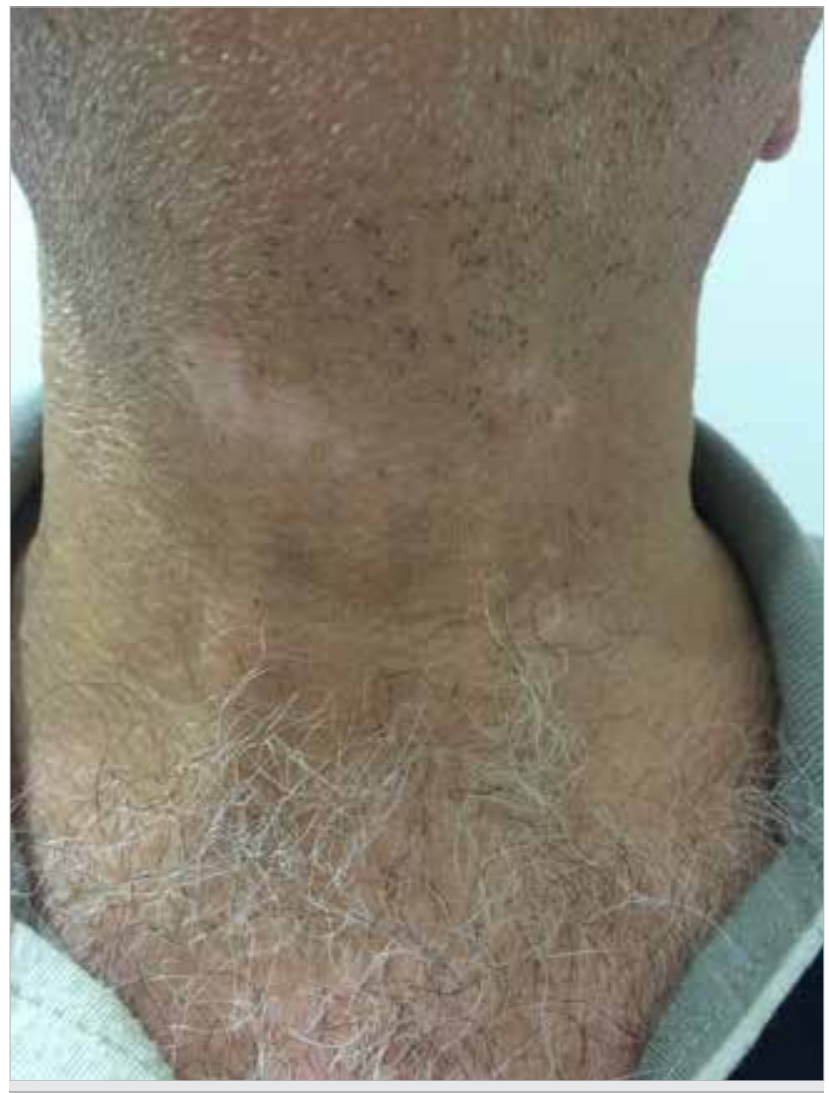

Figure 2. a. Neck before topical corticosteroid treatment

C. spinosa is a plant that grows in Western and Central Asia and in the Mediterranean Basin. It has a strong resistance to harsh environmental conditions and was used as a sweetener in foods and as a diuretic treatment in hypertension in ancient times. It grows in the wilderness in Tunisia from spring until the end of summer (11). Biological studies have shown that it has significant antimicrobial, antioxidant, anti-inflammatory, and antiviral properties. It has been used as a traditional medicine to treat various diseases (12). The presence of alkaloids, lipids, polyphenols, flavonoids, and glucosinolates has been found in studies conducted on the chemical composition of C. spinosa. It has also been reported that C. spinosa is rich in kaempferol, rutin, and flavonoids, such as quercetin and quercetin derivatives (11). It has been shown in studies related to quercetin that it can be a natural and potent inducer of melanogenesis by increasing tyrosinase activity and melanogenesis in human melanocytes (13). In addition, it has cell protective effects against free radicals and active oxygen species. The fact that the increase in free radicals and lipid peroxidation products may also be responsible for depigmentation in vitiligo is due to the etiopathogenetic mechanisms that are suggested. Jimenez-Cervantes et al. reported that $\mathrm{H}_{2} \mathrm{O}_{2}$ inhibits melanogenesis in melanoma cells. Quercetin has also been shown to have suppressive effects on cytotoxicity due to $\mathrm{H}_{2} \mathrm{O}_{2}$ (13). Based on these, a topical cream form has been produced in view of the fact that $C$. spinosa extract may be a treatment alternative in vitiligo. In our study, although repigmentation was observed after treatment in $30 \%$ of the patients who were

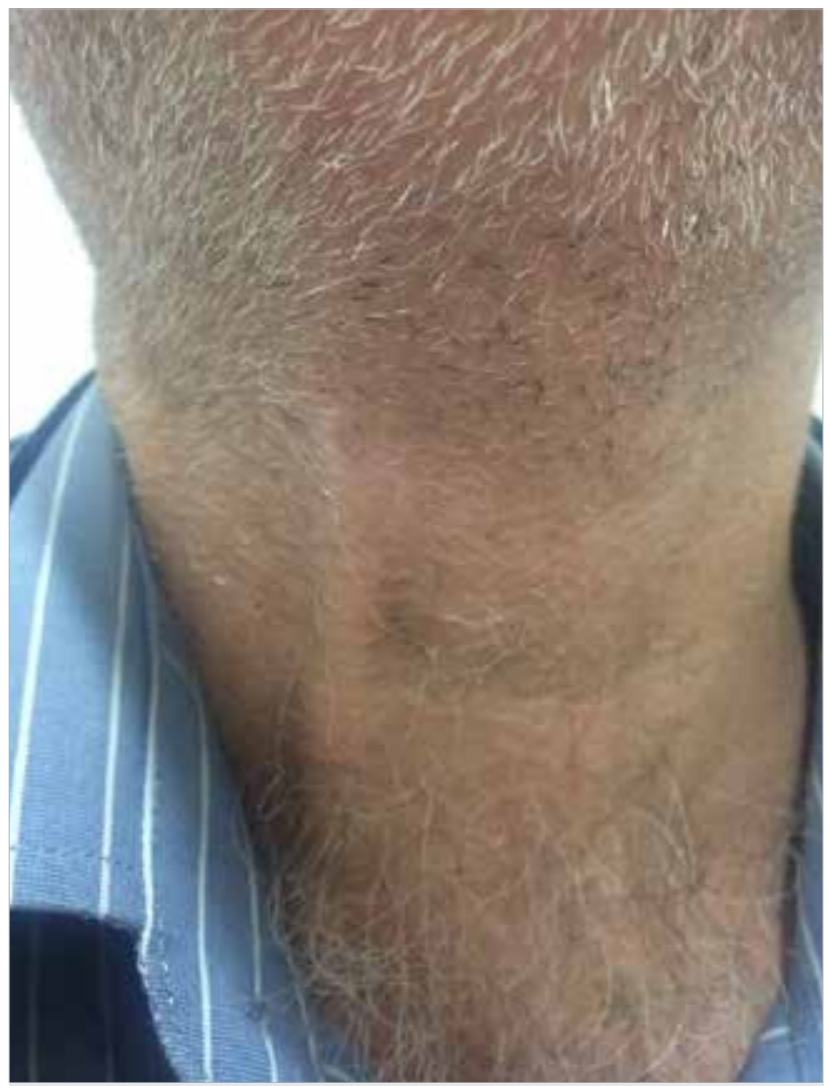

Figure 2. b. Neck after topical corticosteroid treatment

treated with topical C. spinosa in comparison to before (Figure $1 \mathrm{a}-\mathrm{d})$, there was no statistically significant change in their VASIs $(\mathrm{p}>0.05)$ (Table 1$)$.

The use of topical corticosteroids is a treatment method in localized lesions in vitiligo. Its efficacy depends on immunomodulator effects. Although the first choice is accepted in localized vitiligo because of its advantages, such as low cost and ease of use, side effects, such as atrophy, telangiectasia, and striae, limit its use in the long term. Therefore, if there is no response to treatment for 2-3 months in patients receiving topical corticosteroids, treatment should be stopped (14-15). It was shown in some studies that using potent and superpotent steroids provides $75 \%$ repigmentation in approximately $60 \%$ of the patients $(16,17)$. In our study, although repigmentation was observed in $70 \%$ of the patients who received topical corticosteroids after treatment in comparison to before, there was a statistically significant decrease in their VASI $(\mathrm{p}<0.05)$ (Table 1, Figure 2a, b).

When patients using topical C. spinosa were compared with patients using topical corticosteroids, topical corticosteroids were more successful in terms of response to treatment. However, repigmentation was also seen in patients using C. spinosa.

In conclusion, topical corticosteroids were more effective when we compared the efficacy of $C$. spinosa extract, a new option in vitiligo treatment, with topical corticosteroids. However, the fact that repigmentation has been also observed 
in patients using C. spinosa suggests that even if not alone, it is an herbal medicine that can be added to the existing treatments patients receive.

Ethics Committee Approval: Ethics committee approval was received for this study from the Ethics Committee of Bezmialem Vakıf University.

Informed Consent: Written informed consent was obtained from all the patients who participated in this study.

Peer-review: Externally peer-reviewed.

Author Contributions: Concept - N.E., F.P.C., A.G.B., D.B.Ö., Ö.S., N.O.; Design - N.E., F.P.C., A.G.B., D.B.Ö., Ö.S., N.O.; Supervision - N.E., F.P.C., A.G.B., D.B.Ö., Ö.S., N.O.; Resources - N.E., F.P.C., A.G.B., D.B.Ö.; Materials - N.E., F.P.C., A.G.B., D.B.Ö.; Data Collection and/or Processing - N.E., F.P.C., A.G.B., D.B.Ö., Ö.S., N.O.; Analysis and/or Interpretation - N.E., F.P.C., A.G.B., D.B.Ö.; Literature Search - N.E., F.P.C., A.G.B., D.B.Ö.; Writing Manuscript - N.E., F.P.C., A.G.B., D.B.Ö.; Critical Review - N.E., F.P.C., A.G.B., D.B.Ö., Ö.S., N.O.

Conflict of Interest: No conflict of interest was declared by the authors.

Financial Disclosure: The authors declared that this study has received no financial support.

\section{References}

1. Jean-Paul Ortonne. Vitiligo and other disorders of hypopigmentation. In: Bolognia JL, Jorizzo JL, Rapini RP, editors. Dermatology. 1st edition. St. Louis: Mosby; 2003: 947-55.

2. Taieb A. Intrinsic and extrinsic pathomechanisms in vitiligo. Pigment Cell Res 2000; 14: 41-7. [CrossRef]

3. Denli Y. Vitiligo. Dermatoloji'de. 3. Baskı. Ed Tüzün Y, Gürer MA, Serdaroğlu S ve ark. İstanbul, Nobel Tip Kitapevi, 2008; 1465-90.

4. Demirel BG, Borlu M. Vitiligo Tedavisi ve Yeni Tedavi Yaklaşımları. Turk J Dermatol 2010; 4: 33-9.

5. Tamesis ME, Morelli JG. Vitiligo treatment in childhood: a state of the art review. Pediatr Dermatol 2010; 27: 437-45. [CrossRef]

6. Camacho F, Mazuecos J. Oral and topical L-phenylalanine, clobetasol propionate, and UVA/sunlight-a new study for the treatment of vitiligo. J Drugs Dermatol 2002; 1: 127-31.

7. De Leeuw J, van der Beek N, Maierhofer G, Neugebauer WD. A case study to evaluate the treatment of vitiligo with khellin encapsulated in L-phenylalanin stabilized phosphatidylcholine liposomes in combination with ultraviolet light therapy. Eur J Dermatol 2003; 13: 474-77.

8. Dell'Anna ML, Mastrofrancesco A, Sala R. Antioxidants and narrow band-UVB in the treatment of vitiligo: a double-blind placebo controlled trial. Clin Exp Dermatol 2007; 32: 631-36. [CrossRef]

9. Giovanni E Orecchia. Alternative therapies for vitiligo. In: Hann SK, Nordlund JJ, editors. Vitiligo. 1st edition. Oxford: Blackwell Science Ltd 2000; 223-24.

10. Sethi S, Mahajan BB, Gupta RR, Ohri A. Comparative evaluation of the therapeutic efficacy of dermabrasion, dermabrasion combined with topical 5\% -fluorouracil cream, and dermabrasion combined with topical placentrex gel in localized stable vitiligo. Int J Dermatol 2007; 46: 875-79. [CrossRef]

11. Matsuyama K, Villareal MO, El Omri A, Han J, Kchouk ME, Isoda H. Effect of Tunisian Capparis spinosa L. extract on melanogenesis in B16 murine melanoma cells. J Nat Med 2009; 63: 468-72. [CrossRef]

12. Tlili N, Elfalleh W, Saadaoui E, Khaldi A, Triki S, Nasri N. The caper (Capparis L.): Ethnopharmacology, phytochemical and pharmacological properties. Fitoterapia 2011; 82: 93-101. [CrossRef]

13. Nagata H, Takekoshi S, Takeyama R, Homma T, Yoshiyuki Osamura R. Quercetin Enhances Melanogenesis By Increasing the Activity and Synthesis of Tyrosinase in Human Melanoma Cells and in Normal Human Melanocytes. Pigment Cell Res 2004; 17: 66-73. [CrossRef]

14. Mahmoud BH, Hexsel CL, Hamzavi IH. An update on new and emerging options for the treatment of vitiligo. Skin Therapy Lett 2008; 13: 1-6.

15. Kwinter J, Pelletier J, Khambalia A, Pope E. High-potency steroid use in children with vitiligo: a retrospective study. J Am Acad Dermatol 2007; 56: 236-41. [CrossRef]

16. Ezzedine K, Lim HW, Suzuki T, Katayama I, Hamzavi I, Lan CC, et al. Vitiligo Global Issue Consensus Conference Panelists. Revise classification/nomenclature of vitiligo and related issues: the Vitiligo Global Issues Consensus Conference. Pigment Cell Melanoma Res 2012; 25: E1-13. [CrossRef]

17. Taieb A, Alomar A, Böhm M, Dell'anna ML, De Pase A, Eleftheriadou V, et al. Vitiligo European Task Force (VETF); European Academy of Dermatology and Venereology (EADV); Union Europe' enne des Médecins Spé cialistes (UEMS). Guidelines for the management of vitiligo: the European Dermatology Forum consensus. Br J Dermatol 2013; 168: 5-19. [CrossRef] 